\title{
CLUF: a Neural Model for Second Language Acquisition Modeling
}

\author{
Shuyao Xu \\ Singsound Inc. \\ Beijing, China \\ xushuy@ singsound.com
}

\author{
Jin Chen \\ Singsound Inc. \\ Beijing, China \\ chenjin@singsound.com
}

\author{
Long Qin \\ Singsound Inc. \\ Beijing, China \\ qinlong@singsound.com
}

\begin{abstract}
Second Language Acquisition Modeling is the task to predict whether a second language learner would respond correctly in future exercises based on their learning history. In this paper, we propose a neural network based system to utilize rich contextual, linguistic and user information. Our neural model consists of a Context encoder, a Linguistic feature encoder, a User information encoder and a Format information encoder (CLUF). Furthermore, a decoder is introduced to combine such encoded features and make final predictions. Our system ranked in first place in the English track and second place in the Spanish and French track with an AUROC score of $0.861,0.835$ and 0.854 respectively.
\end{abstract}

\section{Introduction}

Education systems that can adapt to the presenting of educational materials according to students' personal learning needs have great potential. Specifically, in the area of second language learning, we try to predict whether the learning materials are too easy or too hard for language learners. Therefore, we study the Second Language Acquisition Modeling (SLAM) task to build a model of the language learning process.

Bayesian Knowledge Tracing (BKT) (Corbett and Anderson, 1994; Pardos and Heffernan, 2010; Pelánek, 2017) that models students' knowledge over time is a well-established problem. It takes a Hidden Markov Model (HMM) with binary hidden states to represent knowledge acquisition for each concept separately. BKT had been successfully applied to subjects like mathematics and programming, where a limited number of concepts can be predefined. However, in language learning, it's difficult to define a small number of concepts, especially when the vocabulary size increases over time. Deep Knowledge Tracing (DKT) (Piech et al., 2015; Wilson et al., 2016) is a recent implementation of knowledge tracing which uses Recurrent Neural Networks (RNNs) to model student's learning trace. Although RNNs and its commonly used variants, such as Gated Recurrent Units (Cho et al., 2014) and Long Short-Term Memory (LSTM) (Hochreiter and Schmidhuber, 1997), are capable of exploring dynamic temporal behavior for a time sequence, it's hard to model extremely long learning history that can range over months even years. Half-life Regression (Settles and Meeder, 2016) is a novel approach for the SLAM task, which combines a psycholinguistic model of human memory with modern machine learning techniques. It had demonstrated state-ofart performance for predicting student recall rates.

Mapping symbols, such as characters or words, into a continuous space is a popular method in natural language processing (Hinton, 1986; Mikolov et al., 2013; Pennington et al., 2014; Mikolov et al., 2017). It achieved remarkable success in many tasks, for example, neural language modeling (Bengio et al., 2003; Collobert and Weston, 2008; Mikolov et al., 2010), machine translation (Sutskever et al., 2014; Bahdanau et al., 2015), text classification (Lai et al., 2015; Zhang et al., 2015; Conneau et al., 2017), sentiment analysis (dos Santos and Gatti, 2014; Poria et al., 2015) and machine reading comprehension (Xiong et al., 2017; $\mathrm{Hu}$ et al., 2017). In this work, we introduce a similar neural approach for the SLAM task, where we use neural encoders to extract features from each exercise as well as metadata about student and session. To be specific, we build a Context encoder, a Linguistic feature encoder, a User information encoder and a Format information encoder (CLUF) to calculate high-level representations from characters, words, part-of-speech (POS) labels, syntactic dependency labels, user id and country, exercise type, client, etc. 


\begin{tabular}{llllccc}
\hline Track & Set & Users & Exercises & $\begin{array}{c}\text { Unique } \\
\text { Tokens }\end{array}$ & $\begin{array}{c}\text { Positive } \\
\text { Ratio }(\%)\end{array}$ & $\begin{array}{c}\text { OOV Ratio } \\
(\%)\end{array}$ \\
\hline \multirow{3}{*}{ en_es } & Train & 2593 & 824012 & 1967 & 12.6 & - \\
& Dev & 2592 & 115770 & 1839 & 14.3 & 3.4 \\
& Test & 2593 & 114586 & 1879 & - & 4.5 \\
\hline \multirow{3}{*}{ es_en } & Train & 2643 & 731896 & 2525 & 14.1 & - \\
& Dev & 2640 & 96003 & 2353 & 15.7 & 7.6 \\
& Test & 2641 & 93145 & 2459 & - & 10.0 \\
\hline \multirow{3}{*}{ fr_en } & Train & 1213 & 326792 & 1941 & 16.2 & - \\
& Dev & 1206 & 43610 & 1671 & 17.6 & 7.1 \\
& Test & 1206 & 41753 & 1707 & - & 5.9 \\
\hline
\end{tabular}

Table 1: The SLAM dataset statistics

\section{Dataset}

The Duolingo SLAM dataset (Settles et al., 2018) is organized into three language tracks:

- en_es: English learners (who already speak Spanish)

- es_en: Spanish learners (who already speak English)

- fr_en: French learners (who already speak English)

According to Table 1, most tokens (more than $80 \%)$ are perfect matches and are given the label 0 for "OK". Tokens that are missing or spelled incorrectly (ignoring capitalization, punctuation, and accents) are given the label 1 denoting a mistake. Across the three language tracks, en_es has the lowest positive ratio, while es_en has the highest out-of-vocabulary (OOV) ratio.

Table 2 shows the features provided with the SLAM dataset. In our system, we used all features except the morphology features and syntactic dependency edges, as we did not get any improvement during experiments. Perhaps it is because that the neural networks already encoded similar information from characters, words and their syntactic dependency labels.

\section{Method}

We used in total four encoders to model the students' learning behavior. Inputs to these encoders are embeddings learned from one-hot representations of raw features. The context encoder consists of a character level LSTM encoder and a word level LSTM encoder. The linguistic feature encoder is also a LSTM model, where POS and syn-

\begin{tabular}{l|l}
\hline Category & Features \\
\hline Context & word surface form \\
\hline Linguistic & $\begin{array}{l}\text { part of speech } \\
\text { morphology features } \\
\text { syntactic dependency edges } \\
\text { syntactic dependency labels }\end{array}$ \\
\hline User & $\begin{array}{l}\text { user id } \\
\text { countries } \\
\text { days in course }\end{array}$ \\
\hline Format & $\begin{array}{l}\text { client } \\
\text { session type } \\
\text { exercise format } \\
\text { response time }\end{array}$ \\
\hline
\end{tabular}

Table 2: Features provided with the SLAM task

tactic dependency embedding are concatenated together and then fed into a multilayer LSTM unit. At last, user encoder and format encoder are both fully-connected neural networks. The user encoder takes account of user id, users' nationality and other user related information, while the format encoder encodes exercise format, session type, client type and time used for the exercise. The decoder combines the outputs of these encoders and then makes predictions through a sigmoid unit.

\subsection{Context Encoder}

The context encoder operates at both the word level and the character level. The word level encoding is capable of capturing better semantics and longer dependency than the character level encoding. But learning new words is a key part in language learning. By modeling the character sequence, we may be able to learn certain word 


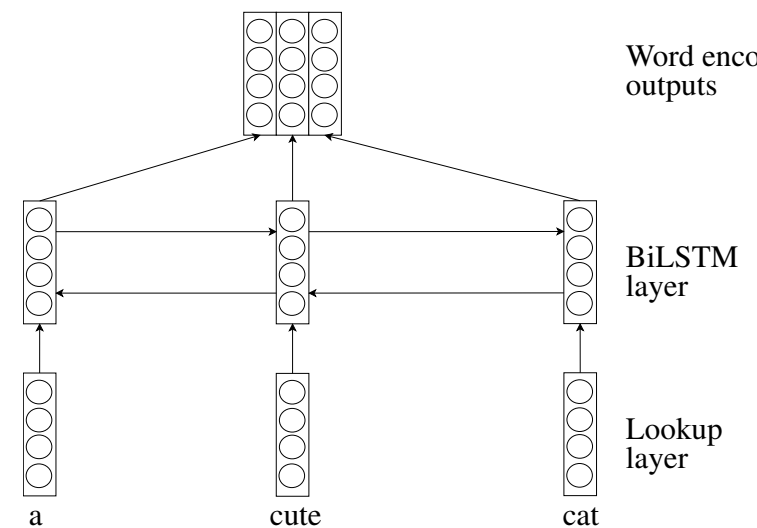

Figure 1: The Word Level Context Encoder

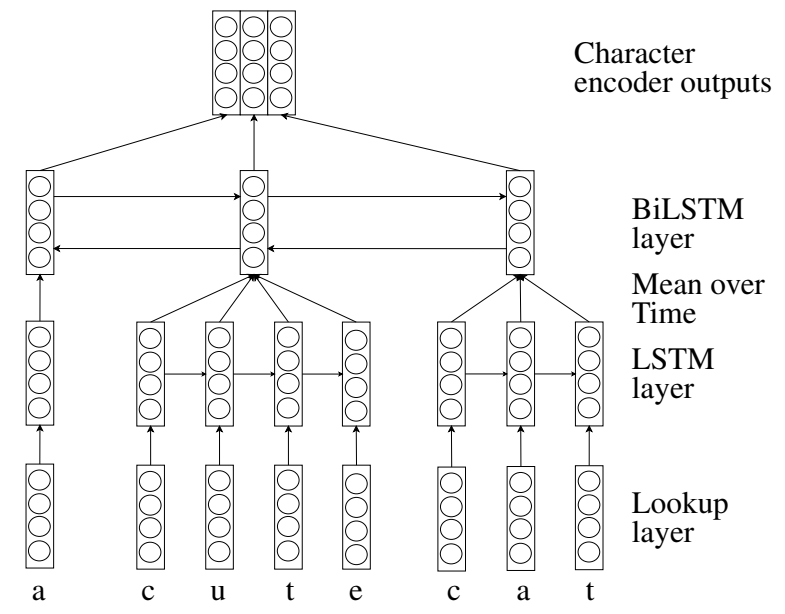

Figure 2: The Character Level Context Encoder

The mean-over-time (MoT) layer takes $H_{w_{t}}$ as inputs

$$
h_{w_{t}}=\frac{1}{M} \sum_{i=1}^{M} h_{i}^{K_{1}} \text {, }
$$

Then the outputs of the MoT layer $\left(h_{w_{1}}, h_{w_{2}}, \ldots\right.$, $h_{w_{N}}$ ) are inputs to a Bidirectional LSTM model,

$$
\begin{aligned}
\hat{f}_{t}^{j} & =\operatorname{LSTM}\left(\hat{f}_{t-1}^{j}, \hat{f}_{t}^{j-1}\right) \\
\hat{b}_{t}^{j} & =\operatorname{LSTM}\left(\hat{b}_{t+1}^{j}, \hat{b}_{t}^{j-1}\right) \\
\hat{g}_{t} & =\left[\hat{f}_{t}^{K_{2}}, \hat{b}_{t}^{K_{2}}\right],
\end{aligned}
$$

where $K_{2}$ is the number of layers of the BiLSTM, $j=1,2, \ldots, K_{2}$.

The final outputs of the context encoder are computed as:

$$
O=\left(o_{1}, o_{2}, \ldots, o_{N}\right),
$$

where $o_{t}=g_{t}+\hat{g}_{t}$.

\subsection{Linguistic Feature Encoder}

The linguistic feature encoder is also a LSTM model. Similar to the context encoder, we trained embedding representations of the POS labels and the syntactic dependency labels. The POS embeddings and syntactic dependency embeddings are concatenated together and then fed into a LSTM unit,

$$
\begin{aligned}
l_{t}^{0} & =\left[\operatorname{pos}_{t}, \operatorname{dep}_{t}\right] \\
l_{t}^{j} & =L S T M\left(l_{t-1}^{j}, l_{t}^{j-1}\right) \\
L & =\left(l_{1}^{K_{3}}, l_{2}^{K_{3}}, \ldots, l_{N}^{K_{3}}\right),
\end{aligned}
$$
$j=1,2, \ldots, K_{1}$. 
where pos $_{t}$ is the POS embedding of word $w_{t}$ and $d e p_{t}$ is the syntactic dependency label embedding of word $w_{t} . j$ is the layer index, and we have $K_{3}$ layers in this LSTM unit.

\subsection{User Encoder}

The user encoder is a one-layer fully-connected feedforward network. The encoder takes user metadata as inputs

$$
\begin{aligned}
& \mu^{0}=[u, s, \text { days }] \\
& \mu^{1}=\tanh \left(W_{\mu} \cdot \mu^{0}+b_{\mu}\right),
\end{aligned}
$$

where $u$ is the embedding of the user id, $s$ is the embedding of the user's nationality and days is the time since the student started learning this language. $W_{\mu}, b_{\mu}$ are trained network parameters. We used the tanh activation function for the user encoder.

\subsection{Format Encoder}

Similar to the user encoder, the format encoder is also a one-layer fully-connected feedforward network. The inputs are format, session, client, and the response time,

$$
\begin{aligned}
& f^{0}=[\text { format }, \text { session }, \text { client }, \text { time }] \\
& f^{1}=\tanh \left(W_{f} \cdot f^{0}+b_{f}\right)
\end{aligned}
$$

where $W_{f}, b_{f}$ are trainable parameters.

\subsection{Decoder}

The decoder takes the outputs $\left(\mathrm{O}, \mathrm{L}, \mu^{1}, f^{1}\right)$ of the context encoder, linguistic encoder, user encoder and format encoder as inputs. The prediction for word $w_{t}$ in the given sequence $\left(w_{1}, w_{2}, \ldots, w_{N}\right)$ is computed as

$$
\begin{aligned}
\nu & =\sigma\left(W_{\nu} \cdot\left[\mu^{1}, f^{1}\right]+b_{\nu}\right) \\
\gamma_{t} & =\sigma\left(W_{\gamma} \cdot\left[l_{t}^{K_{3}}, o_{t}\right]+b_{\gamma}\right) \\
p_{t} & =\sigma\left(W_{p} \cdot\left(\nu \odot \gamma_{t}\right)+b_{p}\right),
\end{aligned}
$$

where $W_{\nu}, b_{\nu}, W_{\gamma}, b_{\gamma}, W_{p}$, and $b_{p}$ are trainable parameters. For decoding, we used the sigmoid activation function $\sigma$.

\subsection{Training}

The model is trained to minimize the following loss function

$$
\begin{aligned}
\text { Loss }=-\frac{1}{N} \sum_{t=1}^{N} & \left(\alpha y_{t} \cdot \log \left(p_{t}\right)+\right. \\
& \left.(1-\alpha)\left(1-y_{t}\right) \cdot \log \left(1-p_{t}\right)\right),
\end{aligned}
$$

\begin{tabular}{l|lr}
\hline Team & AUROC & F1 \\
\hline SanaLabs & 0.861 & 0.561 \\
our model & 0.861 & 0.559 \\
alexrich & 0.859 & 0.468 \\
Masahiro & 0.848 & 0.476 \\
zz & 0.846 & 0.414 \\
Cam & 0.841 & 0.479 \\
btomosch & 0.829 & 0.424 \\
LambdaLearning & 0.821 & 0.389 \\
nihalnayak & 0.821 & 0.376 \\
$\ldots$ & $\ldots$ & $\ldots$ \\
baseline & 0.774 & 0.190 \\
\hline
\end{tabular}

Table 3: Results of the en_es track.

\begin{tabular}{l|lr}
\hline Team & AUROC & F1 \\
\hline SanaLabs & 0.838 & 0.530 \\
our model & 0.835 & 0.524 \\
alexrich & 0.835 & 0.420 \\
Masahiro & 0.824 & 0.439 \\
zz & 0.818 & 0.390 \\
Cam & 0.807 & 0.435 \\
btomosch & 0.803 & 0.375 \\
LambdaLearning & 0.801 & 0.344 \\
Grotoco & 0.791 & 0.452 \\
$\ldots$ & $\ldots$ & $\ldots$ \\
baseline & 0.746 & 0.175 \\
\hline
\end{tabular}

Table 4: Results of the es_en track.

where $\alpha$ is the hyper parameter to balance the negative and positive samples and $y_{t}$ is the label of the time step $t$. In our experiment, we set $\alpha$ to 0.7 .

\section{Experiments and Results}

\subsection{Experiments}

We considered the words that appear less than five times in the training data as unknown token. For students with more than one nationality, only the first one was used.

The embedding size was set to 100 , and the Dropout (Srivastava et al., 2014) regularization was applied, where the dropout rate was set to 0.5. We used the Adam optimization algorithm (Kingma and $\mathrm{Ba}, 2014$ ) with a learning rate of 0.001 . The word level context encoder was a twolayer Bidirectional LSTM. The character level context encoder had one LSTM layer for encoding each word and three Bidirectional LSTM layers above the MoT layer. Furthermore, the linguistic 


\begin{tabular}{l|lr}
\hline Team & AUROC & F1 \\
\hline SanaLabs & 0.857 & 0.573 \\
our model & 0.854 & 0.569 \\
alexrich & 0.854 & 0.493 \\
zz & 0.843 & 0.487 \\
Masahiro & 0.839 & 0.502 \\
Cam & 0.835 & 0.508 \\
btomosch & 0.823 & 0.442 \\
LambdaLearning & 0.815 & 0.415 \\
Grotoco & 0.813 & 0.502 \\
$\ldots$ & $\ldots$ & $\ldots$ \\
baseline & 0.771 & 0.281 \\
\hline
\end{tabular}

Table 5: Results of the fr_en track.

\begin{tabular}{c|ccc}
\hline Term & en_es & es_en & fr_en \\
\hline Relative impr (\%) & 11.24 & 11.93 & 9.72 \\
\hline
\end{tabular}

Table 6: The relative improvement over the baseline

encoder was a two-layer LSTM. Both of the user encoder and format encoder were one-layer fullyconnected feedforward networks.

\subsection{Results}

The evaluation metrics for the SLAM task were the Area Under the Receiver Operation Characteristic (AUROC) curve and the F1 score.

As provided in Table 3, Table 4 and Table 5, our model achieved the AUROC score of 0.861, 0.835, and 0.854 and the F1 score of $0.559,0.524$ and 0.569 for the en_es, es_en, and fr_en track, respectively. We ranked in first place in the en_es track and second place in the es_en and fr_en track.

Table 6 shows that CLUF gained significant improvements on all tracks compared to the baseline model. The improvement on the en_es and es_en track were close, while the improvement on the fr_en track was a bit lower. We think this is because the fr_en (327k exercises) track has much less training data than the en_es (824k exercises) and es_en (732k exercises) track.

\subsection{Discussion}

Our intuition behind CLUF is to factorize raw features into four independent parts: 1) word surface form models the word formation rules; 2) the linguistic encoder is to provide linguistic and syntactic dependency information; 3) the user part explores students' second language acquisition skills

\begin{tabular}{l|lr}
\hline Model & AUROC & F1 \\
\hline CLUF & 0.846 & 0.554 \\
LUF & 0.775 & 0.446 \\
CUF & 0.843 & 0.552 \\
CLF & 0.813 & 0.501 \\
CLU & 0.779 & 0.467 \\
\hline
\end{tabular}

Table 7: Encoder analysis. LUF has no context encoder; CUF has no linguistic encoder; CLF has no user encoder; CLU is the model without format encoder.

over time; 4) the format encoder measures the difficulty level of different exercises on various clients.

Table 7 shows the performance of our CLUF model when excluding one of the context, linguistic, user and format encoder. We can see that the performance drops substantially if we don't use the contextual or format features. On the other hand, excluding the linguistic features does not affect the performance much. At last, we can achieve fairly good performance even if we don't use any user information.

\section{Conclusion}

We presented a neural network based model, CLUF, for the SLAM task. We encoded the contextual, linguistic, user and format features separately. Our system achieved one of the best results in this task. Moreover, our CLUF model was language invariant, as it performed approximately equally well across three language tracks. We further explored how effective each encoder was. We found that the context encoder was the most effective one, while the linguistic encoder was the least effective one.

\section{Acknowledgments}

We thank Duolingo and Educational Testing Service for organizing this novel and interesting task and releasing the SLAM dataset.

\section{References}

Dzmitry Bahdanau, Kyunghyun Cho, and Yoshua Bengio. 2015. Neural machine translation by jointly learning to align and translate. international conference on learning representations.

Yoshua Bengio, Réjean Ducharme, Pascal Vincent, and Christian Jauvin. 2003. A neural probabilistic lan- 
guage model. Journal of machine learning research, 3(Feb):1137-1155.

Kyunghyun Cho, Bart Van Merriënboer, Dzmitry Bahdanau, and Yoshua Bengio. 2014. On the properties of neural machine translation: Encoder-decoder approaches. arXiv preprint arXiv:1409.1259.

Ronan Collobert and Jason Weston. 2008. A unified architecture for natural language processing: Deep neural networks with multitask learning. In Proceedings of the 25th international conference on Machine learning, pages 160-167. ACM.

Alexis Conneau, Holger Schwenk, Loïc Barrault, and Yann Lecun. 2017. Very deep convolutional networks for text classification. In Proceedings of the 15th Conference of the European Chapter of the Association for Computational Linguistics: Volume 1, Long Papers, volume 1, pages 1107-1116.

Albert T Corbett and John R Anderson. 1994. Knowledge tracing: Modeling the acquisition of procedural knowledge. User modeling and user-adapted interaction, 4(4):253-278.

Geoffrey E Hinton. 1986. Learning distributed representations of concepts. In Proceedings of the eighth annual conference of the cognitive science society, volume 1, page 12. Amherst, MA.

Sepp Hochreiter and Jürgen Schmidhuber. 1997. Long short-term memory. Neural computation, 9(8):1735-1780.

Minghao Hu, Yuxing Peng, and Xipeng Qiu. 2017. Reinforced mnemonic reader for machine comprehension. CoRR, abs/1705.02798.

Diederik P Kingma and Jimmy Ba. 2014. Adam: A method for stochastic optimization. arXiv preprint arXiv:1412.6980.

Siwei Lai, Liheng Xu, Kang Liu, and Jun Zhao. 2015. Recurrent convolutional neural networks for text classification. In $A A A I$, volume 333 , pages $2267-$ 2273.

Tomas Mikolov, Edouard Grave, Piotr Bojanowski, Christian Puhrsch, and Armand Joulin. 2017. Advances in pre-training distributed word representations. arXiv preprint arXiv:1712.09405.

Tomáš Mikolov, Martin Karafiát, Lukáš Burget, Jan Černockỳ, and Sanjeev Khudanpur. 2010. Recurrent neural network based language model. In Eleventh Annual Conference of the International Speech Communication Association.

Tomas Mikolov, Ilya Sutskever, Kai Chen, Greg S Corrado, and Jeff Dean. 2013. Distributed representations of words and phrases and their compositionality. In C. J. C. Burges, L. Bottou, M. Welling, Z. Ghahramani, and K. Q. Weinberger, editors, $A d$ vances in Neural Information Processing Systems 26, pages 3111-3119. Curran Associates, Inc.
Zachary A Pardos and Neil T Heffernan. 2010. Modeling individualization in a bayesian networks implementation of knowledge tracing. In International Conference on User Modeling, Adaptation, and Personalization, pages 255-266. Springer.

Radek Pelánek. 2017. Bayesian knowledge tracing, logistic models, and beyond: an overview of learner modeling techniques. User Modeling and UserAdapted Interaction, 27(3-5):313-350.

Jeffrey Pennington, Richard Socher, and Christopher Manning. 2014. Glove: Global vectors for word representation. In Proceedings of the 2014 conference on empirical methods in natural language processing (EMNLP), pages 1532-1543.

Chris Piech, Jonathan Bassen, Jonathan Huang, Surya Ganguli, Mehran Sahami, Leonidas J Guibas, and Jascha Sohl-Dickstein. 2015. Deep knowledge tracing. In C. Cortes, N. D. Lawrence, D. D. Lee, M. Sugiyama, and R. Garnett, editors, Advances in Neural Information Processing Systems 28, pages 505-513. Curran Associates, Inc.

Soujanya Poria, Erik Cambria, and Alexander Gelbukh. 2015. Deep convolutional neural network textual features and multiple kernel learning for utterance-level multimodal sentiment analysis. In Proceedings of the 2015 conference on empirical methods in natural language processing, pages 2539-2544.

Cicero dos Santos and Maira Gatti. 2014. Deep convolutional neural networks for sentiment analysis of short texts. In Proceedings of COLING 2014, the 25th International Conference on Computational Linguistics: Technical Papers, pages 69-78.

B. Settles, C. Brust, E. Gustafson, M. Hagiwara, and N. Madnani. 2018. Second language acquisition modeling. In Proceedings of the NAACL-HLT Workshop on Innovative Use of NLP for Building Educational Applications (BEA). ACL.

Burr Settles and Brendan Meeder. 2016. A trainable spaced repetition model for language learning. In Proceedings of the 54th Annual Meeting of the Association for Computational Linguistics (Volume 1: Long Papers), volume 1, pages 1848-1858.

Nitish Srivastava, Geoffrey Hinton, Alex Krizhevsky, Ilya Sutskever, and Ruslan Salakhutdinov. 2014. Dropout: A simple way to prevent neural networks from overfitting. The Journal of Machine Learning Research, 15(1):1929-1958.

Ilya Sutskever, Oriol Vinyals, and Quoc V Le. 2014. Sequence to sequence learning with neural networks. In Z. Ghahramani, M. Welling, C. Cortes, N. D. Lawrence, and K. Q. Weinberger, editors, $A d$ vances in Neural Information Processing Systems 27, pages 3104-3112. Curran Associates, Inc. 
Kevin H Wilson, Xiaolu Xiong, Mohammad Khajah, Robert V Lindsey, Siyuan Zhao, Yan Karklin, Eric G Van Inwegen, Bojian Han, Chaitanya Ekanadham, Joseph E Beck, et al. 2016. Estimating student proficiency: Deep learning is not the panacea. In In Neural Information Processing Systems, Workshop on Machine Learning for Education.

Caiming Xiong, Victor Zhong, and Richard Socher. 2017. Dynamic coattention networks for question answering. international conference on learning representations.

Xiang Zhang, Junbo Zhao, and Yann LeCun. 2015. Character-level convolutional networks for text classification. In Advances in neural information processing systems, pages 649-657. 